\title{
Chemical Industry with Biomass as Raw Material
}

\author{
Yunshen Zhang \\ Department of Petrochemical Engineering, Puyang Vocational and Technical College, puyang \\ Henan, P. R. China 457000
}

Keywords: biomass; raw materials; chemical industry

\begin{abstract}
In the current social economic development circumstances, people's daily production and life are increasingly dependent on energy resources. However, because coal, oil and so on are non-renewable resources in real life, in this case, In order to ensure that China can achieve long-term development, we need to continue to study the new renewable resources to replace non-renewable resources.Biomass is an important material to replace non renewable resources, such as oil, coal and other non renewable resources which found in long-term studies, and as the basis for the establishment of the chemical industry is called biomass chemical industry. This paper mainly analyzes the necessity of the development of bio chemical industry, and the current status and future development trend of biomass chemical industry are studied, in order to provide reference for the successful development of biomass chemical industry.
\end{abstract}

With the continuous development of social economy, the chemical industry has gradually become an important manifestation of social civilization and development, but as far as the current situation is concerned, people in the production and life of the most dependent resources are almost non-renewable resources, once resource depletion, human development will also be a serious threat. At the same time, coal, oil and other resources because of its non-degradable, so after use is bound to cause pollution of the environment, which can be seen, the use of coal, oil and other resources in the future development of society not only face resource depletion, environmental factors are also important factors that affect the healthy development of human society. In this case, it is necessary to study and find the sustainable development of energy resources and environment in a timely manner. The emergence of biomass resources has been effective. Because the energy itself from nature, and are renewable resources, the following specific to the raw materials for chemical and chemical analysis.

\section{First, the importance of the development of the chemical industry as raw materials}

Biomass resources are considered to be an important material to replace oil, coal and other resources, mainly from three aspects of analysis. First of all, the development of biomass as raw materials, chemical and chemical industry can effectively reduce emissions. This resource itself has the potential to develop low-emission fuels that can effectively reduce carbon dioxide emissions in the transport sector. While biomass as raw material can be carried out cleaning fluid, textile and plastic production, so that people can reduce the use of carbon in life. In addition, the development of biomass as raw materials, chemical and chemical industry, can effectively reduce the development of China's dependence on fossil fuels. From the current development of China, regardless of the production structure or consumption structure, coal energy in which occupy an important position, and oil, natural gas and other energy also has a direct impact on people's lives[1]. And in the process of social development, due to the growing demand for energy, natural gas and other high-quality resources in which the proportion is declining, and because of the imbalance in the distribution of energy itself and the economic development of different regions, The current energy structure is also gradually changing, in which case if you can achieve the biomass as raw material chemical and chemical development, can effectively improve the current energy structure in China, especially for rural areas, which itself has a Rich in biomass energy, if we can do to local conditions for energy access, can effectively reduce our non-renewable energy needs. Finally, the 
development of chemical raw materials to the chemical raw materials to promote the development of China's economy has a positive effect, but also to promote the healthy development of China's ecological environment. Biomass energy is an important part of energy, and it occupies an important position in it. From the point of view of long-term development, biomass resources are renewable, and the pollution to environment is relatively small. This kind of energy is different from our country's current The concept of social development is fully consistent.

\section{Second, biomass Chemical Technology}

\section{1, the application of macromolecular materials}

Biomass itself is composed of many macromolecules closely, but in the actual application of whether to carry out the separation and then degradation or direct degradation, it becomes a problem of using this material. If you choose to be separated, and then degradation, it will produce a number of relatively high molecular weight compounds, and then can achieve its fine degradation, but in order to achieve this goal, faced with an important question is how these Macromolecules for complete separation [2]. If it is directly degraded, it is thermo-chemical decomposition of its final degradation of the molecular weight of the mixture is relatively small, and then use. This approach does not require the separation of macromolecules, but requires the separation of complex degradation of small molecules. Researchers through long-term study found that the separation of macromolecules is relatively good way, because if the macromolecule material can be put directly into the actual use, can effectively avoid the occurrence of polymer polymerization, and in large Molecular separation, the final product degradation, relative to its structure will be more simple, but in this way the use of biomass materials, you need to cellulose, lignin and hemicellulose and other large Molecular separation, which is currently the development of biomass as raw materials to face an important problem, but also lead to the paper industry, the root causes of environmental pollution. Then in the actual biomass to raw materials for the development of chemical and chemical industry, in order to make it better into the use, you need to conduct in-depth research, first of all, the structure of biomass in the relationship between the macromolecule is In-depth research, clear interaction between macromolecules [3]. In addition, the use of physical and chemical, biotechnology to macromolecules for a thorough and efficient separation, and can ensure that can effectively reduce the pollution of the environment. However, the current research situation, the problem has not been effectively resolved, so the actual use of biomass energy, almost all of them are selected to be degraded into small molecule mixture.

\section{2, Thermochemical conversion technology}

Thermochemical conversion is also a technology of biomass chemical industry. The transformation process can be divided into gasification, pyrolysis and direct liquefaction. In the process of pyrolysis and direct liquefaction, the boundary between the two stages is not obvious. During the thermal conversion process, if the effective control of the relevant conversion conditions can be achieved, the syngas of combustible gas can be obtained, and then the combustion gas can be obtained by a certain amount of combustion gas, The synthesis gas can be converted to dimethyl ether and methanol. Methanol is an important raw material in the chemical industry, can be used directly as a fuel, but also with other chemical materials for some of the synthesis of compounds. In addition, the conversion of biomass to chemical fuels and synthetic products is the way the biomass is liquefied or pyrolyzed after the bio-oil will be obtained, and then transform the biomass oil or separation, and thus the fuel and chemicals synthesis. Biomass can also be used in the synthesis of hydrogen from the biomass in the synthesis of hydrogen, you can use the thermochemical or biological method, in the actual study, you can use biomass to produce hydrogen on the basis of biological carbon Base fertilizer development, the final formation of the product is urea and ammonium bicarbonate. 


\section{3, Chemical hydrolysis}

Chemical hydrolysis is another important way of transforming biomass. In the initial process of chemical hydrolysis, sugars and lignins are produced, and then they are further transformed to give different miscellaneous compounds. Usually most of the biomass is cellulose. The cellulose is mainly composed of glucose units in accordance with the $\beta-1,4$ to form a macromolecular link, by hydrolysis can be broken down into glucose, glucose decomposition can be obtained after levulinic acid, which is a chemical industry in a Important raw materials, through the corresponding reaction mode can be different organic compounds. Glucose itself is also a chemical raw materials, in different chemical reactions can be obtained under acid, ketone and alcohol compounds, the transformation can be obtained after the olefin, acid, ester and polymer.

\section{Third, the application of biotechnology and environmental benefits}

\section{1, The application of biotechnology}

Biotechnology plays an important role in the conversion of biomass into synthetic chemicals. In practical research, biotechnology has been able to obtain the corresponding research results, mainly because the conversion rate is quite high, and the condition comparison mild. In the development of biomass chemical and chemical industry, the combination of membrane technology and bio-fermentation technology based on the emergence of genetic engineering, to provide a new direction of development. Acetone, butadiene, butanol, glycerol, pyridine, propylene, ethylene, acetaldehyde and propane are already available from the development of biomass chemistry and chemical engineering, Acrylic acid can also be obtained if allowed. In the development of biomass chemical industry, the use of biomass fermentation for the production of hydrogen is a very important research, mainly because hydrogen from the biomass in the system, in the future has a good development prospects, the use of This technology for biomass conversion of raw materials, the development of clean energy has important significance. The use of biotechnology in a certain amount of conditions can also be more advanced ketones, alcohols and aldehydes, etc., in the actual transformation process, the first need for biomass utilization of lime for processing, and then combined with the fermentation method, you can To obtain 3-pentanol and pentanone and other substances.

\section{2, The environmental benefits of biomass applications}

Biomass as raw material chemistry is an important direction of human society to achieve long-term development, is also a new research direction, the fundamental purpose of this chemical is the chemical reaction process and the effective design of the product, so that it can in the During the reaction, as well as after the separation and use of the entire process, as far as possible to reduce the pollution of humans and the environment. The current research includes greening of raw materials, greening of synthesis methods, greening of chemical processes, greening of reaction reagents and substituting solvents, and the economy of atoms in chemical reactions. Biomass as a product of nature, its survival and recycling in a way that can be fully in the natural cycle of effective treatment, and can effectively reduce the pollution of the environment, therefore, in the actual study, the biomass can The identity of the green raw material replaces the chemicals in the chemical industry in the past. Of course, in order to make the chemical green, not only need to green raw materials, but also in the chemical transformation process to achieve green, which requires the actual study, the chemical replaced by biomass, and then the Green transformation process, but also to ensure that the final product is environmentally friendly features ${ }^{[4]}$. Because most of the biomass structure is cellulose, cellulose can be derived after the chemical raw materials, so in order to effectively achieve the transformation and utilization of biomass, the first need to cellulose in the corresponding medium But because of the molecular chain of cellulose contains hydrogen bonds, so seriously hampered the dissolution of cellulose. It was found that cellulose could be dissolved in room temperature ionic liquid and microwave assisted. Solvent-free derivatization of cellulose is 
also a more important way, with the help of microwave, can make cellulose crystallize to achieve phosphorylation, and then can get cellulose phosphate. As an important component of biomass, lignin is also an important subject in the field of chemical engineering. In the present application, the paper industry will cause serious pollution to the environment. The reason is that there is no effective conversion to the lignin, and the result of the direct discharge is obtained. If it is recovered and then mixed with the polyethylene oxide, the carbon fiber can be obtained after carbonization or spinning.

\section{Fourth, the development trend of biomass chemical industry}

In the current process of social and economic development, non-renewable resources have become unhealthy resources in people's normal life. In the event of an energy crisis, it is bound to affect the development and survival of human society. In order to promote human society to obtain longDevelopment, the future must be the use of energy from non-renewable resources to convert renewable resources. At present, researchers have paid attention to this issue. Biomass chemical and chemical industry has a bright future in the future. The biomass energy to achieve. Replacing coal, oil and other resources, but also can effectively reduce the chemical industry on environmental pollution, which will be from the industrial, energy, economic and environmental aspects to achieve healthy development model.

\section{Concluding remarks}

In summary, the biomass chemical work is a new discipline, now has achieved certain results, the reason why the emergence of this discipline, mainly because in the development process, the demand for resources more and more, but the current In the energy structure, the demand is the largest fossil non-renewable resources, in which case, the human demand for energy is increasing, if you can not develop to replace these energy materials, human survival and development will face Serious threat. Biomass chemical chemistry can be achieved primarily by replacing the chemical raw material with biomass and greening the energy conversion process to ensure that the resulting product can be degraded in nature, by which the energy structure can be effectively Adjust and reduce the pollution of the chemical industry on the environment, and ultimately can promote the socio-economic, chemical energy and the ecological environment of the common development.

\section{References}

[1] Bai Jing, Zhang Lu, Fang Shuqi, Chen Junying, Chang Chun, Cheng Xiaohui, Yang Fuying, Liang Tengbo.Progress in the research of food chemical products based on sugar-based biomass production [J]. Progress in chemical industry, 2015 (1).

[2] Xie Zaiku, Liu Zhicheng, Wang Yangdong.Technology Innovation and Prospect of Petrochemical Industry Facing Resources and Environment [J]. SCIENCE CHINA: Chemistry, $2014(9)$.

[3] Ai Ning, Ji Weirong, Ruan Huimin, Xiang Bin, Xiao Chun, Gao Jianrong.Exploration and practice of the training model of biomass engineering talents [J], Higher Education of Chemical Engineering, 2011 (4).

[4] He Yufeng, Qian Wenzhen, Wang Jianfeng, Xiong Yubing, Song Pengfei, Wang Rongmin. Review of high value-added reuse of waste biomass materials [J]. Transactions of the Chinese Society of Agricultural Engineering, 2016 (15). 